\title{
Efecto del asiento basculante en el tiempo productivo: estudio piloto
}

\section{Effect of seat tilt motion in the productive time: pilot study}

\author{
Jenny Rodríguez-García ${ }^{1}$, Fernanda Maradei ${ }^{2}$, Javier Castellanos-Olarte ${ }^{3}$ \\ ${ }^{1}$ Grupo de Investigación en Ergonomía, Producto y Significado - EPS, Escuela de Diseño Industrial, Universidad Industrial de \\ Santander UIS, Colombia. Email: jk.rodriguezgarcia@gmail.com \\ ${ }^{2}$ Grupo de Investigación en Ergonomía, Producto y Significado - EPS, Escuela de Diseño Industrial, Universidad Industrial de \\ Santander UIS, Colombia. Email: mafermar@uis.edu.co \\ ${ }^{3}$ Universidad Pontificia Bolivariana, Colombia. Email: javier.castellanos@upb.edu.co
}

RECIBIDO: Enero 22, 2017. ACEPTADO: Marzo 31, 2017. Versión FinAL: Mayo 10, 2017.

\begin{abstract}
RESUMEN
Existe evidencia que asocia el dolor lumbar con la postura sedente. Para contrarrestar estos efectos, se ha estudiado los beneficios de bascular el asiento en actividades de conducción, sin embargo se ha encontrado que no existen estudios en trabajo de oficina. Objetivo: Evaluar la influencia del asiento basculante en la percepción de dolor lumbar. Materiales y métodos: se plantearon dos tratamientos: a) asiento con basculación y b) asiento sin basculación; estudiados en cuatro tareas de oficina: leer, escribir a mano, digitar y navegar en internet. Como respuesta se midió la actividad muscular del tronco, la postura de la espalda, el tiempo y el número de errores. Resultados: En las tareas de digitar, navegar en internet y leer existe una diferencia significativa, es decir que la basculación influye en el comportamiento postural y en el tiempo productivo. Conclusiones: Estudiar variables como la presión del asiento para encontrar mayor evidencia.
\end{abstract}

PALABRAS CLAVE: Dolor lumbar, Oficina, Productividad.

\begin{abstract}
Background: There is some evidence that involves the low back pain with sitting posture. To counteract these effects, it has been considered the seat tilt motion only in drivers activities, however it has been found a lack of studies that relates it with office work. Objective assess the influence of seat tilt motion on the perception of lumbar pain. Materials and methods: two treatments raised were a seat: a) with tilt motion and b) without tilt motion; studied in four office tasks: reading, writing, typing and surfing the internet. As study responses were raised muscular activity, back posture, productive time and number of errors. Results: Only in typing, surfing the internet and reading tasks there are a significant differences, that means that the seat tilt motion influence in the postural evaluation and productive time. Conclusions: to assess other variables as seat pressure to find more evidence.
\end{abstract}

KEYWORDS: Lumbar pain, Office, Productivity.

\section{INTRODUCCIÓN}

Los trabajadores de oficina mantienen posturas estáticas en largos periodos de tiempo debido a la naturaleza de las tareas a desarrollar [1]. De acuerdo con esto, las tareas con mayor penosidad en el trabajo de oficina son leer, escribir a mano, navegar en internet y digitar [2]. Se ha podido evidenciar que la introducción intensiva de la informática en el trabajo de oficina ha modificado las actividades desarrolladas por los trabajadores, ocasionando la manifestación de diferentes trastornos musculo-esqueléticos (TME) por la necesidad de permanecer en el puesto de trabajo de manera continua [3] y en postura sedente [4]. El dolor lumbar (DL) es el TME con mayor prevalencia a nivel mundial [5] [6] [7]. En el contexto colombiano se reporta como la segunda patología con mayor prevalencia en los trabajadores, interfiriendo en su desempeño laboral [8]. Es así, que se han considerado intervenciones ergonómicas en el espacio físico de los conductores de vehículos, 
específicamente asientos basculantes que reducen la carga estática de compresión sobre los discos, mejorando la nutrición [9], encontrando que este tipo de modificaciones reducen la percepción de incomodidad debida al dolor lumbar [10]. No obstante la revisión realizada en el marco de este proyecto, encontró que existe poca evidencia en actividades de oficina, asimismo tampoco se conoce los efectos de la basculación en la productividad. De esta manera, este estudio, busca responder la pregunta ¿En qué medida el uso de una silla con asiento basculante en postura sedente, influye en la realización de cuatro tareas de oficina?

\section{MATERIALES Y MÉTODOS}

\subsection{Aprobación ética}

Esta investigación fue aprobada por el Comité de ética de la Universidad Industrial de Santander CEINCI, conforme a las guías contenidas en la declaración de Helsinki y el reporte de Belmont para la investigación con humanos. Adicionalmente, de acuerdo a la Resolución 008430 del 1993 del Departamento de Salud de Colombia, esta investigación está clasificada con riesgo mínimo. Finalmente, los participantes antes de cada prueba firmaron el consentimiento informado, en donde se aclara en la totalidad la ejecución del experimento.

\subsection{Participantes}

Fueron escogidas 8 trabajadoras voluntarias de la Universidad Industrial de Santander, ya que las mujeres tienen más riesgo de TME en comparación con los hombres [11]. Estas participantes estaban en un estado nutricional óptimo con un Indice de Masa Corporal (IMC) entre los 19 y 25 [12] [13].

\subsection{Variable independiente y tratamientos}

- Silla con asiento basculante (CB), con un mecanismo ubicado en la parte inferior del asiento que permite el movimiento de $10^{\circ}$ hacia adelante y $5^{\circ}$ hacia atrás, desde el plano sagital.

- Silla sin basculación (SB) con asiento fijo.

\subsection{Descripción de las variables dependientes}

- Actividad muscular. Estudiada mediante electromiografía (EMG), ubicando el sensor Sx320 de Biometrics Ltd Uk en el vientre de los músculos: erector lumbar derecho de la espalda RES (ver Figura 1) y músculo recto mayor del abdomen RA (ver Figura 2).
- Postura. Estudiada con un goniómetro de espalda SG150B Biometric Ltd Uk (ver Figura $1)$.

- Tiempo productivo. Para tomar estos tiempos se usó un cronómetro, tomando el momento en el que se inició cada tarea y el momento en el que se finaliza la misma.

- Errores. Definidos de acuerdo a cada una de las tareas, en donde en la tarea de digitar y escribir a mano, basándose en el estudio realizado por Werth y BabskiReeves [14], se tomaron como errores la inserción de una letra extra en una palabra, inclusión de una letra en una palabra, ca pitalizar una palabra sin necesidad o no capitalizarla en el caso de ser necesario, sustituir una letra errada en el lugar correcto de esta y trasponer 2 letras consecutivas. En la tarea de leer se definieron como errores la lectura de más de dos veces de una palabra, devolverse más de dos veces en un párrafo, dejar de leer por más de $30 \mathrm{~s}$, releer todo el contenido y la subvocalización. En la tarea de leer se tomaron la posición de las letras y la migración de palabras [15]; y por último, en la tarea de navegar en internet fueron la navegación con más de 3 clicks por acción a realizar, la navegación en páginas diferentes a la estipulada o que no tengan relación con la tarea a desarrollar, salirse de la tarea 2 veces y devolverse reiterativamente (más de 2 veces) a un punto de trabajo.

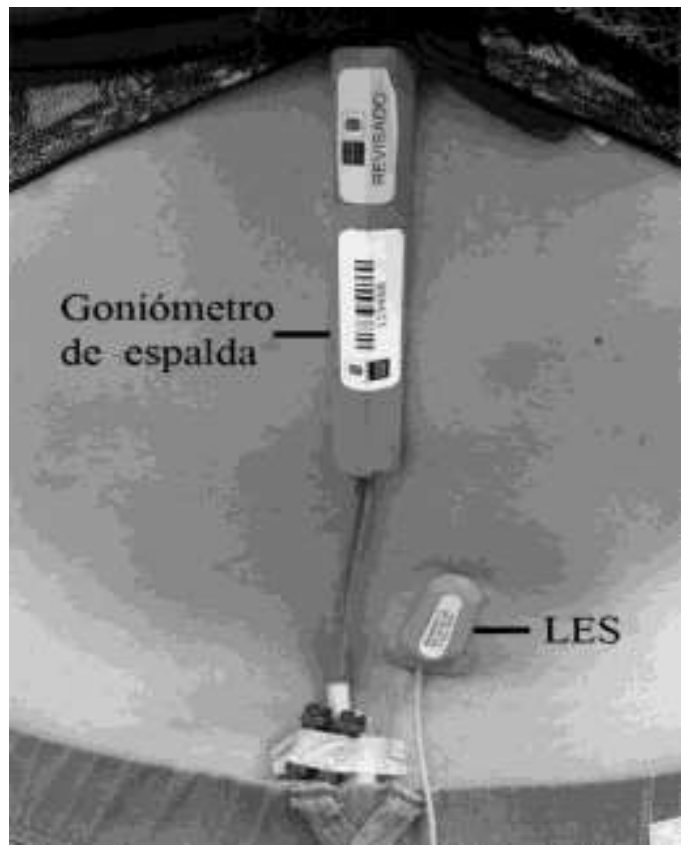

Figura 1. Sensor Sx320 ubicado en el vientre del músculo erector lumbar derecho de la espalda LES y el goniómetro de espalda SG150B. Fuente. Elaboración propia. 


\subsection{Tiempo productivo}

La definición de los tiempos productivos de cada tarea, se realizó mediante una prueba con 15 participantes para su estandarizaron como elemento guía y de control en la medición del efecto con cada uno de los tratamientos [16], en una silla estándar de oficina (sin basculación y sin inclinación del asiento). Es así que los tiempos productivos, en donde se ejecutan las tareas, teniendo en cuenta las fallas de diseño y tiempos suplementarios fueron: 1379 segundos digitando, 1177 segundos escribiendo a mano, 1361 segundos navegando en internet y 1195 segundos leyendo. De esta manera, se tomaron cada uno de estos tiempos productivos estandarizados (TE) como el 100\% de ejecución de la tarea y se estimarán en los resultados como tiempos adicionales (+) o tiempo restante (-).

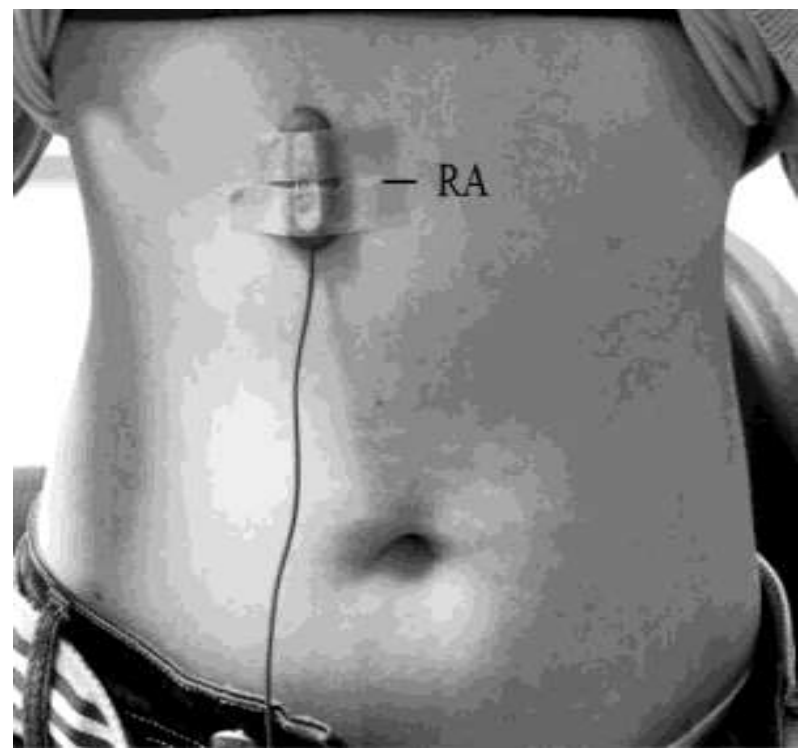

Figura 2. Sensor Sx320 ubicado en el vientre del músculo recto mayor derecho del abdomen RA. Fuente. Elaboración propia.

\subsection{Procedimiento}

Se asignaron aleatoriamente los tratamientos (CB y SB) y las tareas a desarrollar por cada participante. Cada tratamiento se estudió dos veces en cada participante, teniendo como tiempo total máximo de ejecución de la prueba media jornada laboral $(4 \mathrm{~h})$.

Se realizó la lectura y firma del consentimiento informado para así empezar a adherir cada uno de los equipos sobre la piel de las participantes. El primer día se tomó la Máxima Contracción Voluntaria (MVC), de los músculos erector lumbar derecho de la espalda (LES) y recto mayor derecho del abdomen (RA) para el posterior procesamiento de datos.
Teniendo claro todo lo anterior, se le indicaba a la participante que debía sentarse para ajustar las condiciones del asiento respecto al puesto de trabajo y así se daba inicio a la ejecución de la primera tarea. Entre cada una de las tareas, la participante debía levantarse y realizar un ciclo de ejercicios espinales de 25 segundos, para permitir la relajación de la espalda, mostrados en la Tabla 1.

Tabla 1. Ejercicios de relajación de espalda después de cada tarea.

\begin{tabular}{|l|l|}
\hline $\begin{array}{l}\text { Lumbares y } \\
\text { paravertebrales }\end{array}$ & $\begin{array}{l}\text { Elevar los brazos y las } \\
\text { manos al máximo y } \\
\text { ponerse de puntillas (5s). }\end{array}$ \\
\hline $\begin{array}{l}\text { Dorsales e } \\
\text { intercostales }\end{array}$ & $\begin{array}{l}\text { Con las piernas } \\
\text { ligeramente separadas y } \\
\text { los brazos estirados, rotar } \\
\text { hacia un lado y luego } \\
\text { hacia el otro (10s). }\end{array}$ \\
\hline $\begin{array}{l}\text { Dorsales e } \\
\text { intercostales }\end{array}$ & $\begin{array}{l}\text { Con las piernas } \\
\text { ligeramente separadas y } \\
\text { las manos en la cintura, } \\
\text { inclinar la espalda, los } \\
\text { hombros y la cabeza } \\
\text { hacia atrás (10s). }\end{array}$ \\
\hline
\end{tabular}

\subsection{Análisis estadístico}

El análisis de resultados de este estudio se realizó con el apoyo del programa IBM SPSS Statistics 22 y serán presentados de acuerdo a las 4 tareas planteadas para cada variable de evaluación descrita anteriormente. Todos los análisis se realizaron comparando los dos tratamientos CB y SB. Para los datos con distribución normal se utilizó el análisis de varianza por medio de ANOVA. Se consideró diferencias significancias con $\mathrm{p}$-valor $<0,05$. Asimismo la actividad del músculo erector fue analizada con Chi-cuadrado para datos no paramétricos, se consideró diferencias significativas con p-valor $<0,05$.

\section{RESULTADOS}

\subsection{Actividad muscular}

Mediante el análisis descriptivo de los datos obtenidos en EMG, se encontró que para la tarea digitar la actividad muscular es mayor con el tratamiento CB ( $\mathrm{RA}=0,041$; $\mathrm{LES}=0,0423$ ). En las tareas de escribir a mano y navegar en internet el comportamiento de la actividad muscular en el músculo LES son parecidas, mientras que en el músculo RA es mayor en el tratamiento CB. Por último, la tarea de leer presenta mayores valores en la actividad 
muscular de LES mientras que en RA es la misma para los tratamientos.

Al realizar la prueba Chi-cuadrado en la comparación de la distribución observada de los datos $(\mathrm{p}<0,05)$, no existe una diferencia significativa entre la actividad muscular de los dos tratamientos de acuerdo a las tareas a desarrollar, como se muestra en la Tabla 2.

Tabla 2. Comparación de EMG por medio de Chi-Cuadrado. Los datos corresponden a la significancia total (p-valor) tomada del programa IBM SPSS Statistics 22.

\begin{tabular}{|l|l|l|l|l|}
\hline Músculo & Digitar & $\begin{array}{l}\text { Escribir } \\
\text { a mano }\end{array}$ & $\begin{array}{l}\text { Navegar } \\
\text { en } \\
\text { internet }\end{array}$ & Leer \\
\hline LES & 0,382 & 0,313 & 0,313 & 0,45 \\
\hline RA & 0,45 & 0,45 & 0,616 & 0,249 \\
\hline
\end{tabular}

Fuente. Elaboración propia.

\subsection{Postura}

En el análisis descriptivo de los ángulos adoptados en flexión, desviación lateral derecha y desviación lateral izquierda, mostrado en la Tabla 3 , se evidencia que en las tareas digitar, escribir a mano y navegar en internet es menor la flexión del tronco $\mathrm{CB}$ y mayor la desviación lateral derecha $\mathrm{CB}$, en comparación con el tratamiento SB. En la deviación lateral izquierda, las tareas de digitar, escribir a mano y leer, tienen valores muy cercanos, mientras que la tarea de navegar en internet CB presenta menores valores que el tratamiento SB. Por último, la tarea leer, en los dos tratamientos, tienen los ángulos muy similares en tanto en flexión como en desviaciones.

Por otra parte, los ángulos en cada uno de los tratamientos y tareas fueron analizados mediante la prueba de Shapiro-Wilk mostrando una distribución normal ( $p>0,071)$. De esta manera, la comparación de medias a través de ANOVA, ver Tabla 4, encontró que: en la tarea digitar existe diferencias significativas entre los tratamientos $\mathrm{CB}$ y SB en los ángulos de flexión $(\mathrm{p}=0,016)$ y la desviación lateral derecha $(\mathrm{p}=0,030)$, confirmando el análisis descriptivo de los datos. Por otro lado, en la tarea de navegar en internet se comprueba que existe una diferencia significativa en la flexión $(\mathrm{p}=0,003)$ entre los tratamientos CB y SB. Por tanto, se descarta otro tipo de diferencias de los ángulos en la postura adoptaba por las participantes.

\subsection{Tiempo productivo}

De acuerdo a la Tabla 5, se observa que el tiempo productivo es mayor en las tareas de digitar, escribir a mano y leer con el tratamiento $\mathrm{CB}$, por lo cual, los participantes tuvieron tiempos adicionales mayores, haciendo que la ejecución de estas fuera en tiempos mucho más extensos.

Asimismo, el número de errores de las tareas digitar y escribir a mano con este mismo tratamiento es siempre mayor, en comparación con el tratamiento SB. Por otro lado, en la tarea navegar en internet, los dos tratamientos muestran un tiempo menor al TE, pero el tratamiento CB tiene menor porcentaje de tiempo productivo, traducido en un tiempo más corto en la ejecución de la tarea. En esta tarea los errores son considerados iguales, por esto, se realizó la toma del número de clicks en donde el tratamiento $\mathrm{CB}$ presentó el mayor número de estos $\left(n^{\circ}\right.$ clicks $\left.=200,75\right)$ en comparación con el tratamiento sin basculación $\left(\mathrm{n}^{\circ}\right.$ clicks $\left.=186,8\right)$. Por último, en la tarea de leer, el tratamiento SB presenta menor porcentaje de tiempo adicional al TE, pero mayor número de errores.

Tabla 3. Postura promedio en cada tarea para los tratamientos CB y SB.

\begin{tabular}{|l|l|l|}
\hline Digitar & CB (DE) & SB $(\mathrm{DE})$ \\
\hline Flexión & $51,45(11,38)$ & $68,93(16,24)$ \\
\hline DesvLatDere & $4,64(3,87)$ & $1,4(1,2)$ \\
\hline DesvLatIzq & $5,36(4,31)$ & $5,07(3,73)$ \\
\hline
\end{tabular}

\begin{tabular}{|l|l|l|}
\hline $\begin{array}{l}\text { Escribir a } \\
\text { mano }\end{array}$ & $\mathrm{CB}(\mathrm{DE})$ & $\mathrm{SB}(\mathrm{DE})$ \\
\hline Flexión & $52,8(7,76)$ & $64,18(20,17)$ \\
\hline DesvLatDere & $2,87(2,46)$ & $1,93(1,91)$ \\
\hline DesvLatIzq & $7,58(4,94)$ & $8,14(4,61)$ \\
\hline
\end{tabular}

\begin{tabular}{|l|l|l|}
\hline $\begin{array}{l}\text { Navegar en } \\
\text { internet }\end{array}$ & CB (DE) & SB (DE) \\
\hline Flexión & $51,65(4,71)$ & $76,87(17,45)$ \\
\hline DesvLatDere & $5,95(3,10)$ & $2,93(2,89)$ \\
\hline DesvLatIzq & $5,16(4,22)$ & $9,65(5,24)$ \\
\hline
\end{tabular}

\begin{tabular}{|l|l|l|}
\hline Leer & CB $(\mathrm{DE})$ & SB $(\mathrm{DE})$ \\
\hline Flexión & $55,31(7,59)$ & $60,97(17,45)$ \\
\hline DesvLatDere & $4,63(3,85)$ & $5,46(4,55)$ \\
\hline DesvLatIzq & $5,11(3,55)$ & $5,22(3,44)$ \\
\hline
\end{tabular}

DE: Desviación estándar

Fuente. Elaboración propia. 
El tiempo productivo y el número de errores en cada uno de los tratamientos y tareas fueron analizados mediante la prueba de Shapiro-Wilk mostrando una distribución normal $(\mathrm{p}>0,06)$. De esta manera, la comparación de medias a través de ANOVA evidenció que sólo en la tarea leer hay diferencias significativas entre los errores de cada tratamiento, en donde CB presenta menor número de estos $(\mathrm{p}=0,035)$.

Tabla 4. Comparación de medias de postura por medio de ANOVA. Los datos corresponden a la significancia total (pvalor) tomada del programa IBM SPSS Statistics 22.

\begin{tabular}{|l|l|l|l|l|}
\hline Postura & Digitar & $\begin{array}{c}\text { Escribir } \\
\text { a mano }\end{array}$ & $\begin{array}{c}\text { Navegar } \\
\text { en } \\
\text { internet }\end{array}$ & Leer \\
\hline Flexión & $\mathbf{0 , 0 1 6}$ & 0,231 & $\mathbf{0 , 0 0 3}$ & 0,347 \\
\hline $\begin{array}{l}\text { Desv } \\
\text { LatDere }\end{array}$ & 0,03 & 0,804 & 0,078 & 0,796 \\
\hline $\begin{array}{l}\text { Desv } \\
\text { LatIzq }\end{array}$ & 0,724 & 0,558 & 0,094 & 0,632 \\
\hline
\end{tabular}

Fuente. Elaboración propia.

Tabla 5. Tiempos productivos y errores de las 4 tareas en cada tratamiento. Los valores en las casillas de cada tarea para cada tratamiento corresponden al tiempo adicional al 100\% (TE), en donde el porcentaje negativo corresponde a que las tareas se hicieron en menos tiempo al TE.

\begin{tabular}{|l|c|c|c|c|c|}
\hline \multirow{2}{*}{ Tarea } & \multicolumn{2}{|c|}{ CB } & \multicolumn{2}{c|}{ SB } & TE \\
\cline { 2 - 6 } & $\mathbf{t}$ & $\mathbf{e}$ & $\mathbf{t}$ & $\mathbf{e}$ & $\mathbf{t}$ \\
\hline Digitar & $+16 \%$ & 112 & $+11,9 \%$ & 102,3 & 1379 \\
\hline $\begin{array}{l}\text { Escribir } \\
\text { a mano }\end{array}$ & $+12,5 \%$ & 13,3 & $+1,1 \%$ & 11,5 & 1177 \\
\hline $\begin{array}{l}\text { Navegar } \\
\text { en } \\
\text { Internet }\end{array}$ & $-11,1 \%$ & 4 & $-2,5 \%$ & 4,75 & 1361 \\
\hline Leer & $+12,9 \%$ & 12,4 & $+8,4 \%$ & 18,38 & 1195 \\
\hline
\end{tabular}

TE: Tiempo productivo estandarizado (100\%).

Fuente. Elaboración propia.

En el estudio realizado por O'Sullivan et al [17], se encontró que estar sentado en una silla dinámica reducía la flexión lumbar y la activación del músculo iliocostal lumbar torácico en la tarea de digitación. De esta manera, el estudio tuvo los mismos comportamientos en términos de flexión al realizar el análisis postural en todas las tareas. En este caso, la flexión del tronco fue siempre menor al realizar las tareas con la silla $\mathrm{CB}\left(48,8^{\circ}-55,31^{\circ}\right)$ en comparación con SB $\left(60,97^{\circ}-76,87^{\circ}\right)$, con diferencia significativa en las tareas de digitar y navegar en internet. Estos ángulos se encuentran cercanos a la postura neutral, definida entre $\operatorname{los} 45^{\circ}-60^{\circ}$ [18] [19], lo cual indica que hay un aumento de la pendiente del tronco hacia adelante esencial para la estática vertebral y generar estabilidad [20] y que al realizar este tipo de tareas de digitación a alta velocidad esta postura es percibida como la más efectiva por parte de los trabajadores [21] [22]. Cabe resaltar que los ángulos de la flexión encontrados en este estudio del tratamiento SB concuerdan con los encontrados en la tarea de digitar realizado por otros autores [23].

De estos ángulos se puede llegar a concluir que en el uso de la silla $\mathrm{CB}$ se produce una menor compresión discal [24] específicamente en estas dos tareas, ya que al estar hacia adelante tiene un efecto de protección hacia la presencia del dolor lumbar al mejorar la nutrición de los discos [25], además permite el equilibrio entre los músculos anteriores y posteriores del tronco. En este sentido, la silla favoreció una postura dinámica en los momentos donde se percibió incomodidad [26] [27] [28], dados por la inclinación del tronco hacia adelante, pero que en comparación con el asiento SB, fueron de menor magnitud. De igual manera, en términos de desviación lateral derecha, la única tarea en la que hubo diferencia significativa fue en digitar; a que la mano dominante era la derecha por lo cual adoptaban esta postura de manera estática usando rangos pequeños de movimiento lumbar (30\% del rango total de movimiento) [29]. Asimismo se sugiere estudios futuros que determinen si existen factores de incomodidad que condicionaron esta postura.

Por otra parte, Muñoz et al [19] definen que la inclinación del tronco tiene una influencia en la actividad de los músculos, encontrando que en la zona lumbar es de $0,08^{\circ}$ con una inclinación del asiento de $-15^{\circ}$, de $0,14^{\circ}$ para asientos con $15^{\circ}$ y de $0,09^{\circ}$ con asientos sin inclinación. De acuerdo a esto, los resultados encontrados en este estudio fueron valores de actividad muscular menores, tanto en los asientos CB y SB. También, al no encontrar diferencias significativas entre los tratamientos, se pudo concluir que la actividad muscular en los dos tratamientos es la misma para las cuatro tareas planteadas.

En términos de tiempos productivos y error, se encontró diferencia significativa entre tratamientos en la tarea de leer, en donde las participantes tuvieron un mayor porcentaje de tiempo productivo en el tratamiento CB. Con relación al TE $(+12,9 \%$ del $\mathrm{TE})$, el número de errores disminuyó significativamente, esto puede ser dado por que no se presentó fatiga, a pesar de que la tarea requiriera de mayor atención visual en la pantalla [30], afectando de forma positiva en la calidad de ejecución. De esta manera, se pudo evidenciar que es necesario, al momento de evaluar el trabajo de oficina, así como todo tipo de trabajo, la productividad ya que es uno de los indicadores importantes que mide el desempeño de una 
buena gestión [31] al momento de realizar un trabajo específico.

Finalmente se evidenció que las personas ajustaron las sillas al iniciar cada una de las tareas, pero al usar la silla CB tenían una actitud de rechazo hacia ésta debido a la incompatibilidad con su modelo mental de uso[32] [33], en donde al cambiar el entorno del puesto de trabajo, debe darse un acompañamiento continuo con retroalimentación constante [31] ya que al iniciar la prueba se mencionó la posibilidad de bascular el asiento pero no tuvo un impacto en la recordación de las participantes. En este sentido Alnaser y Wughalter [34] determinaron que los participantes duran bastante tiempo identificando los controles y características de los mecanismos que se ponen a disposición de ellos en los puestos de trabajo, en donde la mayoría de estos llegan a desecharse o al desuso. Además, se pudo confirmar que el cambio de postura depende de la tarea y no del tipo de silla [27] [34].

\section{RECOMENDACIONES}

Se sugiere, en primera medida, la toma de datos con una muestra más grande, dadas las caracteírsticas de las variables de evaluación y tener mayores datos que soporten el comportamiento humano al interacturar con estos tratamientos. También, estudiar la presión del asiento para encontrar mayor evidencia que ayude a entender el comportamiento humano al ejecutar estas cuatro tareas. Por otro parte, desarrollar un sistema basculante que sea autónomo, dado que en este estudio las personas, ya sea por olvido al ejecutar la tarea o percepción del sistema, no hacían uso de este.

\section{REFERENCIAS}

[1] F. Maradei, Z. Galindo, J. Castellanos "Comfort testing in office chair design," Rev. UIS Ingenierías, vol. 16, no. 1, pp. 67-71, Ene - Jun 2017.

[2] F. Maradei, F. Espinel Z. Galindo, "Percepción del dolor lumbar debido al uso de un asiento dinámico en postura sedente prolongada," Rev. de Salud Pública, pp. 412-424, Mar. 2012.

[3] B.A. Ramirez, J.M. Castillo, "El análisis multifactorial del trabajo estático y repetitivo. Estudio del trabajo en actividades de servicio," Rev. Ciencias de la Salud, vol. 7, pp. 65-82, 2009.

[4] W. Marras, W. Karwowski, The occupational ergonomics hand book, Estados Unidos: CRC Press LCC, 1998.
[5] B. Duthey, World Health Organization, Feb, 2013, [En línea]. Disponible en: http://www.who.int/medicines/areas/priority_medicines/ BP6_10malaria.pdf

[6] K. Luoma et al, "Low back pain in relation to lumbar disc degeneration," Spine, vol. 25, pp. 487-492, 2000.

[7] J. Collins \& L. O'Sullivan, "Psychosocial risk exposures and musculoskeletal disorders across workingage males and females," Human Factors and Ergonomics in Manuf. \& Service Industries, vol. 20, pp. 272-286, 2010.

[8] E. Archila, M. Villamizar, J. Jimenez, "El dolor: una perspectiva epidemiológica", Salud UIS, vol. 36, pp. 213,2004

[9] B. Pandurangan et al, "Musculoskeletal computational analysis of the influence of car-seat design/adjustments on long-distance driving fatigue," Intern. Journ. of Ind. Ergonomics, vol. 40, no. 3, pp. 345$355,2010$.

[10] F. Maradei, L. Quintana, J. Castellanos, "Influence of seat tilt motion on the discomfort perception during a simulated driving activity," Work, vol. 56, no. 1, pp. 99110.

[11] L. Karlqvist et al, "Self-reported working conditions of VDU operators and associations with musculoskeletal symptoms: a cross-sectional study focussing on gender differences," Intern. Journ. of Ind. Ergonomics, vol. 30, pp. 277-294, 2002.

[12] F. Maradei, F. Espinel, A. Peña, "Estudio de Valores Antropométricos Para La Región Nororiental Colombiana 2007-2008," Rev. UIS Ingenierías, vol. 7, no. 2, pp. 153-167, Dic. 2008.

[13] F. Maradei, F. Espinel, L. Díaz, "Diseño de una plataforma informática para el almacenamiento y análisis de datos antropométricos," Rev. UIS Ingenierías, vol. 7, no. 1 pp. 41-51, Jun. 2008.

[14] A. Werth, K. Babski-Reeves, "Effects of portable computing devices on posture, muscle activation levels and efficiency," Applied Ergonomics, vol. 45, pp. 16031609, 2014.

[15] R. Tarrasch, Z. Berman, N. Friedmann, "Mindful Reading: Mindfulness Meditation Helps Keep Readers with Dyslexia and ADHD on the Lexical Track," Frontiers in Psychology, vol. 578, no. 7, 2016. 
[16] C. R. Cavassa, Ergonomía y productividad, Ciudad de México D.F., México: Grupo Noriega Editores, Limusa S.A de C.V, 1997.

[17] K. O’Sullivan, "Lumbar posture and trunk muscle activation during a typing task when sitting on a novel dynamic ergonomic chair," Ergonomics, vol. 55, no. 12, pp. 1586-1595, Dec 2012.

[18] M. R. Jouvencel, Ergonomia básica aplicada a la medicina del trabajo, 1st ed. Madrid, España: Ediciones Díaz de Santos, 1994.

[19] F. Munoz, P.R Rougier, "Estimation of centre of gravity movements in sitting posture: application to trunk backward tilt.," Journ. of Biomechanics, vol. 44, no. 9, pp. 1771-5, Jun 2011.

[20] D. B. Chaffin, Gunnar. BJ Andersson, B.J. Martin, Occupational Biomechanics, $4^{\text {th }}$ ed, 2006.

[21] N. Marmaras, D. Nathanael, N. Zarboutis, "The transition from CRT to LCD monitors: Effects on monitor placement and possible consequences in viewing distance and body postures," Intern. Journ. of Ind. Ergonomics, vol. 38, pp. 584-592, 2008.

[22] R. Lueder, K. Noro, "Three myths of ergonomics seating," en Hard Facts about Soft Machines: The Ergonomics of Seating, London, U.K: Taylor and Francis, 1994, pp. 37-46.

[23] C. Sommerich et al, "Effects of notebook computer configuration and task on user biomechanics,productivity,and comfort," Ind. Ergonomics, vol. 30, pp. 7-31, 2002.

[24] L.H. Barrero et al, "Work pattern causes bias in selfreported activity duration: a randomised study of mechanisms and implications for exposure assessment and epidemiology.," Occupational and Environmental Medicine, vol. 66, no. 1, pp. 38-44, Ene 2009.

[25] M. Makhsous et al, "Biomechanical effects of sitting with adjustable ischial and lumbar support on occupational low back pain: evaluation of sitting load and back muscle activity," BMC Musculoskeletal Disorders, vol. 10, p. 17, Feb 2009.

[26] M. Makhsous et al, "The Effect of Chair Designs on Sitting Pressure Distribution and Tissue Perfusion.," Human Factors, vol. 54, no. 6, pp. 1066-1074, 2012.

[27] J. Van Dieen, M. De Looze, V. Hermans. "Effects of dynamic office chairs on trunk kinematics, "Ergonomics, vol. 44, no. 7, pp. 739-750, 2001.
[28] P.J Mork, R.H Westgaard, "Back posture and low back muscle activity in female computer, "Clinical Biomechanics, vol. 24, no. 2, pp. 169-175, 2009.

[29] J.P Callaghan N.M Dunk, "Lumbar spine movement patterns during prolonged sitting differentiate low back pain developers from matched asymptomatic controls"Work, vol. 35, no. 1, pp. 3-14, 2010.

[30] M. F. Maradei-García, F. Espinel-Correal Z. Galindo-Estupiñan, "Percepción del dolor lumbar debido al uso de un asiento dinámico en postura sedente prolongada", Rev. salud pública, vol. 18, no. 3, pp. 412424, Feb. 2016.

[31] G. Mejía, T. Hernández, "Seguimiento De La Productividad En Obra: Técnicas De Medición De Rendimientos De Mano De Obra", Rev. UIS Ingenierías, vol. 6, no. 2, pp. 45-59, Dic. 2007.

[32] M. Karwowski, The occupational ergonomics, hand book. Estados Unidos: CRC Press LCC, 1999.

[33] D. Norman, The psychology of everyday things: Basic books., 1988.

[34] M. Alnaser, E. Wughalterb, "Effect of chair design on ratings of discomfort," Work, vol. 34, pp. 223-234, 2009. 